\title{
Optics
}

\section{Theory of excitons and excitonic quasimolecules formed from spatially separated electrons and holes in quasi - zero - dimensional nanosystems}

\author{
Sergey I. Pokutnyi \\ Chuiko Institute of Surface Chemistry, National Academy of Sciences of Ukraine, 17 General Naumov Str., UA - 03164 Kyiv, Ukraine
}

Email address:

Pokutnyi_Sergey@inbox.ru

\section{To cite this article:}

Sergey I. Pokutnyi. Theory of Excitons and Excitonic Quasimolecules Formed from Spatially Separated Electrons and Holes in Quasi Zero - Dimensional Nanosystems. Optics. Special Issue: Optics and Spectroscopy of the Charge Carriers and Excitons States in Quasi Zero - Dimensional Nanostructures. Vol. 3, No. 6-1, 2014, pp. 10-21. doi: 10.11648/j.optics.s.2014030601.13

\begin{abstract}
The theory of an exciton formed from spatially separated electron and hole (the hole is in the quantum dot volume, and the electron is localized at the outer spherical quantum dot-dielectric matrix interface) is developed within the modified effective mass method. The effect of significantly increasing the exciton binding energy in quantum dots of zinc selenide, synthesized in a borosilicate glass matrix, relative to that in a zinc selenide single crystal is revealed. It was shown that the short-wavelength shift of the peak of the low-temperature luminescence spectrum of samples containing zincselenide quantum dots, observed under the experimental conditions, is caused by quantum confinement of the ground-state energy of the exciton with a spatially separated electron and hole. A review devoted to the theory of excitonic quasimolecules (biexcitons) (formed of spatially separated electrons and holes) in a nanosystem that consists of $\mathrm{ZnSe}$ quantum dots synthesized in a borosilicate glass matrix is developed within the context of the modified effective mass approximation. It is shown that biexciton (exciton quasimolecule) formation is of the threshold character and possible in nanosystem, in with the spacing between the quantum dots surfaces is larger than a certain critical spacing. On the basis of analogy spectroscopy of electronic states of superatoms (or artificial atoms) and individual alkali metal atoms theoretically predicted a new artificial atom, which is similar to the new alkali metal atom.
\end{abstract}

Keywords: Excitons, Modified Effective Mass Method, Exciton Binding Energy, Quantum Dots, Excitonic Quasimolecules, Biexcitons, Spatially Separated Electrons and Holes, Superatoms

\section{Introduction}

At present, quasi-zero-dimensional semiconductor nanosystems consisting of spherical semiconductor nanocrystals, i.e., quantum dots with radii of $a=1-10 \mathrm{~nm}$, containing cadmium sulfide and selenide, gallium arsenide, germanium, silicon, and zinc selenide in their volume, synthesized in a borosilicate glass matrix, attract particular attention due to their unique photoluminescence properties, i.e., the ability to efficiently emit light in the visible or near infrared ranges at room temperature [1-10]. The optical and electro-optical properties of such quasi-zero dimensional nanosystems are controlled to a large extent by the energy spectrum of the spatially confined electron-hole pair (exciton) [4-16].
In most theoretical models for calculating the energy spectra of quasiparticles in quantum dots (QDs), the effective mass approximation is used, which was considered to be applicable to QDs by analogy with bulk single crystals [11-13]. However, the problem of the applicability of the effective mass approximation to the description of semiconductor QDs is still unsolved [4-18].

In [14], a new modified effective mass method was proposed to describe the exciton energy spectrum in semiconductor QDs with radii of $a \approx a_{e x}\left(a_{e x}\right.$ is the exciton Bohr radius in the semiconductor material contained in the QD volume). It was shown that, within a model in which the QD is represented as an infinitely deep potential well, the effective mass approximation can be applied to the description of an exciton in QDs with radii $a$ comparable to the exciton Bohr radius $a_{\mathrm{ex}}$, assuming that the reduced effective exciton mass is a function of the radius $a, \mu=\mu(a)$. 
In the adiabatic approximation and within the modified effective mass method [14], an expression for the binding energy of an exciton whose electron and hole move in the semiconductor QD volume was derived in [15]. In [15], the effect of significantly increasing the exciton-binding energy in cadmium selenide and sulfide QDs with radii $a$, comparable to the exciton Bohr radii aex, relative to the exciton- binding energy in cadmium selenide and sulfide single crystals (by factors of 7.4 and 4.5 , respectively) was also detected.

In the experimental study [7], it was found that excess electrons produced during interband excitation of the cadmium sulfide QD have a finite probability of overcoming the potential barrier and penetrating into the borosilicate glass matrix into which the QD is immersed. In experimental studies [10, 19] (as well as in [7]) of glass samples with cadmium-sulfide and zinc-selenide QDs, it was found that the electron can be localized in the polarization well near the outer QD surface, while the hole moves within the QD volume.

In $[10,19]$, the optical properties of borosilicate glass samples containing QD zinc selenide are experimentally studied. The average radii of such QDs are in the range $a \approx$ $2.0-4.8 \mathrm{~nm}$. In this case, the values of $a$ are comparable to the exciton Bohr radius $a_{e x} \approx 3.7 \mathrm{~nm}$ in a $\mathrm{ZnSe}$ single crystal. At low QD concentrations, when the optical properties of the samples are mainly controlled by those of individual QDs in the borosilicate glass matrix, a shift of the peak of the low temperature luminescence spectrum to the short wavelength region (with respect to the band gap $E_{g}$ of the zinc selenide - single crystal) was observed. The authors of [10] assumed that this shift is caused by quantum confinement of the energy spectra of the electron and hole localized near the spherical surface of the QD. In this case, the following problem remained open: the quantum confinement of which electron and hole states (the hole moving in the QD volume and the electron localized at the outer spherical QD-dielectric matrix interface or the electron and hole localized in the QD volume) caused such a shift of the luminescence spectrum peak?

The use of semiconductor nanosystems as the active region of nanolasers is prevented by the low binding energy of the QD exciton [8, 9, 13]. Therefore, studies directed toward the search for nanostructures in which a significant increase in the binding energy of QD excitons would be observed are of importance.

Currently, the theory of exciton states in quasi- zerodimensional semiconductor nanosystems has not been adequately studied, in particular, no theory exists for an exciton with a spatially separated electron and hole in quasi- zero- dimensional nanosystems. Therefore, in this study, we developed the theory of an exciton formed from spatially separated electron and hole (the hole is in the semiconductor QD volume, and the electron is localized at the outer spherical surface of the QD-dielectric matrix interface) [20 - 22]. It was shown that the short wavelength shift of the peak of the low temperature luminescence spectrum of samples containing zinc-selenide QDs, observed under the experimental conditions of [10], is caused by quantum confinement of the ground state energy of the exciton with a spatially separated electron and hole. The effect of significantly increasing the binding energy of an exciton (with a spatially separated electron and hole) in a nanosystem containing zinc-selenide QDs in comparison with the binding energy of an exciton in a zinc-selenide single crystal (by a factor of 4.1-72.6) was detected [20 22].

In $[10,19]$, a shift of the spectral peak of the lowtemperature luminescence wasobserved also for samples with a QD concentrations from $x=0.003$ to $1 \%$. It was noted $[10,19]$ that, at such a QD content in the samples, one must take into account the interaction between charge carriers localized above the QD surfaces. Therefore, in [23, 24] we develop the theory of a excitonic quasimolecules (biexcitons) (formed from spatially separated electrons and holes) in a nanosystem that consists of ZnSe QDs synthesized in a borosilicate glass matrix.

\section{Exciton Ground - State Energy in the Nanosystem}

Let us consider the simple model of a quasi - zero dimensional system, i.e., a neutral spherical semiconductor QD of the radius $a$, which contains semiconductor material with the permittivity $\varepsilon 2$ in its volume, surrounded by a dielectric matrix with the permittivity $\varepsilon_{1}$. A hole $h$ with the effective mass $m_{h}$ moves in the QD volume, while an electron $e$ with the effective mass $m_{e}{ }^{(1)}$ lies in the matrix $\left(r_{e}\right.$ and $r_{h}$ are the distances from the QD center to the electron and hole). Let us assume that the QD valence band is parabolic. Let us also assume that there is an infinitely high potential barrier at the spherical QD - dielectric matrix interface; therefore, the hole $h$ cannot leave the QD volume and the electron $e$ cannot penetrate into the QD volume in the model under study [20 - 22].

The characteristic dimensions of the problem are the quantities

$$
\mathrm{a}_{\mathrm{h}}=\varepsilon_{2} \hbar^{2} / \mathrm{m}_{\mathrm{h}} \mathrm{e}^{2}, \mathrm{a}_{\mathrm{ex}}=\varepsilon_{2} \hbar^{2} / \mu \mathrm{e}^{2}, \quad \mathrm{a}_{\mathrm{e}}=\varepsilon_{1} \hbar^{2} / \mathrm{m}_{\mathrm{e}}^{(1)} \mathrm{e}^{2},
$$

where $a_{h}$ and $a_{\mathrm{ex}}$ are the hole and exciton Bohr radii in the semiconductor with the permittivity $\varepsilon 2, e$ is the elementary charge, $\mu=m_{e}{ }^{(2)} m_{h} /\left(m_{e}{ }^{(2)}+m_{h}\right)$ is the reduced effective mass of the exciton, $m_{e}{ }^{(2)}$ is the effective mass of an electron in the semiconductor with the permittivity $\varepsilon_{2}$, and $a_{e}$ is the electron Bohr radius in the dielectric matrix with the permittivity $\varepsilon 1$. The fact that all characteristic dimensions of the problem are significantly larger than the interatomic distances $a_{0}$,

$$
a, a_{e}, a_{h}, a_{e x}>a_{0}
$$


allows us to consider the electron and hole motion in the quasi - zero - dimensional nanosystem in the effective mass approximation [11-13].

We analyzed the conditions of carrier localization in the vicinity of a spherical dielectric particle of the radius $a$ with the permittivity $\varepsilon 2$ in [25-27]. There the problem of the field induced by the carrier near a dielectric particle immersed in a dielectric medium with the permittivity $\varepsilon_{1}$ was solved in a final analytical form, and analytical expressions for the potential energy of the interaction of the carrier with the spherical interface of two media are presented.

Solving the Poisson equation with usual electrostatic boundary conditions

$$
\begin{aligned}
& \left.\varphi\left(r^{\prime}, j \mid \boldsymbol{r}, i\right)\right|_{r^{\prime}=a}=\left.\varphi\left(\boldsymbol{r}^{\prime}, j\right)\right|_{r^{\prime}=a} \\
& \left.\varepsilon_{1} \frac{\partial \varphi\left(\boldsymbol{r}^{\prime}, j \mid \boldsymbol{r}, i\right)}{\partial \boldsymbol{r}^{\prime}}\right|_{r^{\prime}=a}=\left.\varepsilon_{2} \frac{\partial \varphi\left(\boldsymbol{r}^{\prime}, j\right)}{\partial \boldsymbol{r}^{\prime}}\right|_{r^{\prime}=a},
\end{aligned}
$$

the potential $\varphi\left(r^{\prime}, j \mid r, i\right) \mid$ at the observation point $r^{\prime}$ in a medium with the permittivity $\varepsilon_{j}$, induced by the charge $e$ at the point $r$ in a medium with the permittivity $\varepsilon_{i}$, can be presented as a sum of the potentials induced by the image point charge $e^{\prime}\left(r_{i j} \mid r\right)$ at the point $r_{i j}=(a / r)^{2} r \delta_{i j}+r(1-\delta i j)$ and the linear distribution with the density $\rho_{i j}(y, r)$ of the image charge along a straight line passing through the center of the dielectric particle with the radius $a$ and the charge at the point $r$ [25-27]:

$$
\varphi\left(\boldsymbol{r}^{\prime}, j \mid \boldsymbol{r}, i\right)=\frac{e}{\varepsilon_{j}\left|\boldsymbol{r}^{\prime}-\boldsymbol{r}\right|}+\frac{e^{\prime}\left(\boldsymbol{r}_{i j} \mid \boldsymbol{r}\right)}{\varepsilon_{j}\left|\boldsymbol{r}^{\prime}-\boldsymbol{r}_{i j}\right|}+\frac{1}{\varepsilon_{j}} \int_{0}^{\infty} \frac{d y \rho_{i j}(y, \boldsymbol{r})}{\left|\boldsymbol{r}^{\prime}-y(\boldsymbol{r} / r)\right|},
$$

where

$$
\begin{gathered}
\mathrm{r}_{11}=\left(a^{2} / r^{2}\right) \boldsymbol{r}, e^{\prime}\left(\mathrm{r}_{11} \mid \mathrm{r}\right)=-\beta(a / r) e, \\
\rho_{11}(y, r)=\beta \alpha\left(a^{2} / r y\right)^{(1-\alpha)}(e / a) \theta\left(\left(a^{2} / r\right)-y\right) ; \\
\mathrm{r}_{22}=\left(a^{2} / r^{2}\right) \mathrm{r}, e^{\prime}\left(\mathrm{r}_{22} \mid \mathrm{r}\right)=\beta(a / r) e, \\
\rho_{22}(y, r)=\beta(1-\alpha)\left(a^{2} / r y\right)^{\alpha}(e / a) \theta\left(y-\left(a^{2} / r\right)\right) ; \\
r_{12}=r, e^{\prime}\left(\mathrm{r}_{12} \mid \mathrm{r}\right)=\beta e \\
\rho_{12}(y, r)=\beta(1-\alpha)(r / y)^{\alpha}(e / r) \theta(y-r) ; \\
r_{21}=r, e^{\prime}\left(r_{12} \mid r\right)=-\beta e, \\
\rho_{21}(y, r)=\beta \alpha(r / y)^{(1-\alpha)}(e / r) \theta(r-y),
\end{gathered}
$$

where $\theta(x)$ is the Heaviside unit-step function,

$$
\beta=\frac{\left(\varepsilon_{2}-\varepsilon_{1}\right)}{\left(\varepsilon_{2}+\varepsilon_{1}\right)}, \alpha=\frac{\varepsilon_{1}}{\left(\varepsilon_{2}+\varepsilon_{1}\right)}
$$

Using expressions (3)-(3d), the energy $U\left(\mathrm{r}_{\mathrm{e}}, \mathrm{r}_{\mathrm{h}}, a\right)$ of the polarization interaction of the electron and hole with the spherical QD-matrix interface at the relative permittivity $\varepsilon$ $=\left(\varepsilon_{2} / \varepsilon_{1}\right) \gg 1$ can be presented as an algebraic sum of the energies of the interaction of the hole and electron with self- $V_{h h^{\prime}}\left(r_{h}, a\right), V_{e e^{\prime}}\left(r_{e}, a\right)$ and "foreign" $V_{e h^{\prime}}\left(r_{e}, r_{h}, a\right), V_{h e^{\prime}}\left(r_{e}\right.$, $\left.r_{h}, a\right)$ images, respectively [15,16,26-28],

$$
\begin{aligned}
U\left(\mathrm{r}_{\mathrm{e}}, \mathrm{r}_{\mathrm{h}}, a, \mathcal{E}\right)= & V_{h h^{\prime}}\left(r_{h}, a, \mathcal{E}\right)+V_{e e^{\prime}}\left(r_{e}, a, \mathcal{E}\right) \\
& +V_{e h^{\prime}}\left(\mathrm{r}_{\mathrm{e}}, \mathrm{r}_{\mathrm{h}}, a, \mathcal{E}\right)+V_{h e^{\prime}}\left(\mathrm{r}_{\mathrm{e}}, \mathrm{r}_{\mathrm{h}}, a, \mathcal{E}\right),
\end{aligned}
$$

where

$$
\begin{gathered}
V_{h h^{\prime}}\left(r_{h}, a, \varepsilon\right)=\frac{e^{2} \beta}{2 \varepsilon_{2} a}\left(\frac{a^{2}}{a^{2}-r_{h}^{2}}+\varepsilon\right), \\
V_{e e^{\prime}}\left(r_{e}, a, \varepsilon\right)=-\frac{e^{2} \beta}{2 \varepsilon_{1} a} \cdot \frac{a^{4}}{r_{e}^{2}\left(r_{e}^{2}-a^{2}\right)}, \\
V_{h e^{\prime}}\left(\mathrm{r}_{\mathrm{e}}, \mathrm{r}_{\mathrm{h}}, a, \varepsilon\right)=\frac{e^{2} \beta}{2 \varepsilon_{2} a} \cdot \frac{a^{2}}{r_{e}\left|\mathrm{r}_{\mathrm{h}}-\left(a / r_{e}\right)^{2} \mathrm{r}_{\mathrm{e}}\right|}, \\
V_{e h^{\prime}}\left(\mathrm{r}_{\mathrm{e}}, \mathrm{r}_{\mathrm{h}}, a, \varepsilon\right)=-\frac{e^{2} \beta}{2 \varepsilon_{1} a} \cdot \frac{a^{2}}{r_{h}\left|\mathrm{r}_{\mathrm{e}}-\left(a / r_{h}\right)^{2} \mathrm{r}_{\mathrm{h}}\right|} .
\end{gathered}
$$

In the studied simple model of a quasi zero dimensional nanostructure within the above approximations and in the effective mass approximation using the triangular coordinate system [14-16], $r_{e}=\left|r_{e}\right|, r_{h}=\left|r_{h}\right|, r=\left|r_{e}-r_{h}\right|$, with the origin at the center of the QD, the exciton Hamiltonian (with a spatially separated hole moving within the QD volume and an electron in the dielectric matrix) takes the form [20 - 22, 29 - 32]:

$$
\begin{gathered}
H\left(\mathrm{r}_{\mathrm{e}}, \mathrm{r}_{\mathrm{h}}, \mathrm{r}, a\right)=-\frac{\hbar^{2}}{2 m_{e}^{(1)}}\left(\frac{\partial^{2}}{\partial r_{e}^{2}}+\frac{2}{r_{e}} \cdot \frac{\partial}{\partial r_{e}}+\frac{r_{e}^{2}-r_{h}^{2}+r^{2}}{r_{e} r} \cdot \frac{\partial^{2}}{\partial r_{e} \partial r}\right)- \\
-\frac{\hbar^{2}}{2 m_{h}}\left(\frac{\partial^{2}}{\partial r_{h}^{2}}+\frac{2}{r_{h}} \cdot \frac{\partial}{\partial r_{h}}+\frac{r_{h}^{2}-r_{e}^{2}+r^{2}}{r_{h} r} \cdot \frac{\partial^{2}}{\partial r_{h} \partial r}\right)-\frac{\hbar^{2}}{2 \mu_{0}}\left(\frac{\partial^{2}}{\partial r^{2}}+\frac{2}{r} \cdot \frac{\partial}{\partial r}\right)+ \\
+V_{e h}(\mathrm{r})+U\left(\mathrm{r}_{\mathrm{e}}, \mathrm{r}_{\mathrm{h}}, a, \varepsilon\right)+V_{e}\left(r_{e}\right)+V_{h}\left(r_{h}\right)+E_{g},
\end{gathered}
$$

where the first three terms are the operators of the electron, hole, and exciton kinetic energy, Eg is the band gap in the semiconductor with the permittivity $\varepsilon_{2}, \mu_{0}=$ $m_{e}^{(1)} m_{h} /\left(m_{e}^{(1)}+m_{h}\right)$ is the reduced effective mass of the exciton (with a spatially separated hole and electron). In the Hamiltonian (10), the polarization interaction energy $U\left(\mathrm{r}_{e}\right.$, $\left.\mathrm{r}_{h}, a, \varepsilon\right)$ (5) is defined by formulas (6)-(9), and the electron-hole Coulomb interaction energy $\operatorname{Veh}(r)$ is described by the formula

$$
V_{e h}(r)=-\frac{1}{2}\left(\frac{1}{\varepsilon_{1}}+\frac{1}{\varepsilon_{2}}\right) \frac{e^{2}}{r}
$$

In the exciton Hamiltonian (10), the potentials

$$
\begin{aligned}
& V_{h}\left(r_{h}\right)= \begin{cases}0, & r_{h} \leq a \\
\infty, & r_{h}>a\end{cases} \\
& V_{e}\left(r_{e}\right)=\infty, \quad r_{e} \leq a
\end{aligned}
$$


describe the quasiparticle motion using the models of an infinitely deep potential well.

As the QD radius $a$ increases (so that $a \gg a_{e x}^{0}$ ), the spherical interface of the two media (QD-matrix) passes to the plane /semiconductor material with the permittivity $\left.\varepsilon_{2}\right\rangle$-matrix interface. In this case, the exciton with the spatially separated electron and hole (the hole moves within the semiconductor material and the electron lies in the borosilicate glass matrix) becomes two-dimensional [20 $-22]$.

The main contribution to the potential energy of the Hamiltonian (10) describing exciton motion in a nanosystem containing a large-radius $\mathrm{QD}, a \gg a_{e x}^{0}$, is made by the electron-hole Coulomb interaction energy $V_{e h}(r)(11)$. The energy of the hole and electron interaction with self- $V_{h h^{\prime}}\left(r_{h}, a, \varepsilon\right)(6), V_{e e^{\prime}}\left(r_{h}, a\right)(7)$ and "foreign" $V_{e h}{ }^{\prime}\left(\boldsymbol{r}_{e}\right.$ $\left.\boldsymbol{r}_{h}, a\right)$ (9), $V_{h e^{\prime}}\left(\boldsymbol{r}_{e}, \boldsymbol{r}_{h}, a\right)$ (8) images make a significantly smaller contribution to the potential energy of the Hamiltonian (10). In the first approximation, this contribution can be disregarded. In this case, only the electron-hole Coulomb interaction energy (11) remains in the potential energy of the Hamiltonian (10) [20 - 22]. The Schrodinger equation with such a Hamiltonian describes a two-dimensional exciton with a spatially separated electron and hole (the electron moves within the matrix, and the hole lies in the semiconductor material with the permittivity $\varepsilon_{2}$ ), whose energy spectrum takes the form [33, 34]:

$$
\begin{gathered}
E_{n}=-\frac{R y_{e x}^{0}}{(n+1 / 2)^{2}}, \\
R y_{e x}^{0}=\frac{\left(\varepsilon_{1}+\varepsilon_{2}\right)^{2}}{4 \varepsilon_{1}^{2} \varepsilon_{2}^{2}}\left(\frac{\mu_{0}}{m_{0}}\right) R y_{0}
\end{gathered}
$$

where $n=0,1,2 \ldots$ is the principal quantum number of the exciton and $\mathrm{Ry}_{0}=13.606 \mathrm{eV}$ is the Rydberg constant. The Bohr radius of such a two- dimensional exciton is described by the formula

$$
\begin{gathered}
a_{e x}^{0}=\frac{2 \varepsilon_{1} \varepsilon_{2}}{\varepsilon_{1}+\varepsilon_{2}} \frac{\hbar^{2}}{\mu_{0} e^{2}}, \quad \begin{array}{l}
\text { (14) } \\
\text { we write the average value of the exciton Hamiltonian (10) } \\
\text { in wave functions (16) in the form }
\end{array} \\
E_{0}(a, \mu(a))=\left\langle\psi_{0}\left(r_{e}, r_{h}, r, a\right)\left|H\left(r_{e}, r_{h}, r, a\right)\right| \psi_{0}\left(r_{e}, r_{h}, r, a\right)\right\rangle \\
=\int_{a}^{\infty} d r_{e} \int_{0}^{a} d r_{h} \int_{r}^{r_{e}+r_{h}} d r r r_{e} r_{h} \psi_{0}\left(r_{e}, r_{h}, r, a\right) H\left(r_{e}, r_{h}, r, a\right) \psi_{0}\left(r_{e}, r_{h}, r, a\right)
\end{gathered}
$$

and the binding energy of the ground state of such a twodimensional exciton, according to (13), is written as

$$
E_{e x}^{0}=-4 R y_{e x}^{0}
$$

The binding energy (15) of the exciton ground state is understood as the energy required for bound electron and hole state decay (in the state with $n=0$ ).

To determine the ground-state energy of an exciton (with a spatially separated electron and hole) in a nanosystem containing QDs of the radius $a$, we use the variational method. When choosing the variational exciton wave function, we use an approach similar to that developed in [14]. Let us write the variational radial wave function of the exciton ground - state ( $1 s$ electron state and $1 s$ hole state) in the nanosystem under study in the form [20 - 22]:

$$
\begin{gathered}
\psi_{0}\left(r_{e}, r_{h}, r, a\right)=A \exp \left(-\frac{\mu(a)}{\mu_{0}} \frac{r}{a_{e x}^{0}}\right) \frac{\left(a^{2}-r_{h}^{2}\right)}{a^{2}} \\
\times \frac{\left(r_{e}^{2}-a^{2}\right)}{a^{2}} \frac{r}{a} \frac{\left|r_{e}-\left(a / r_{h}\right)^{2} r_{h}\right| r_{h}}{a^{2}} \frac{\left|r_{h}-\left(a / r_{e}\right)^{2} r_{e}\right| r_{e}}{a^{2}}
\end{gathered}
$$

Here the coefficient $A$ is determined from the condition of normalization of the exciton wave function (16),

$$
\int_{a}^{\infty} r_{e} d r_{e} \int_{0}^{a} r_{h} d r_{h} \int_{r}^{r_{e}+r_{h}} \psi_{0}^{2}\left(r_{e}, r_{h}, r, a\right) r d r=1,
$$

and the effective reduced exciton mass $\mu(a)$ is the variational parameter.

As the QD radius $a$ increases (so that $a \gg a_{e x}^{0}$ ), a twodimensional exciton is formed in the nanosystem. This factor leads to the fact that the variational exciton wave function (16) contains the Wannier-Mott two-dimensional exciton wave eigenfunction [33, 34]. Furthermore, the polynomials from $r_{e}$ and $r_{h}$ enter the exciton variational function (16), which make it possible to eliminate singularities in the functional $E_{0}(a, \mu(a))$ in the final analytical form.

To determine the exciton ground-state energy $E 0(a, \varepsilon)$ in the nanosystem under study by the variational method,
The dependence of the energy $E_{0}(a)$ of the exciton ground state $\left(n_{e}=1, l_{e}=m_{e}=0 ; n_{h}=1, l_{h}=m_{h}=0\right),\left(n_{e}, l_{e}\right.$, $m_{e}$ and $n_{h}, l_{h}, m_{h}$ are the principal, orbital, and magnetic quantum numbers of the electron and hole, respectively) on the QD radius $a$ is calculated by minimizing the functional $E_{0}(a, \mu(a))(17)$

$$
\frac{\partial E_{0}(a, \mu(a))}{\partial \mu(a)}=F(\mu(a), a)
$$

Without writing cumbersome expressions for the first derivative of the functional $\partial E_{0}(a, \mu(a)) / \partial \mu(a)=F(\mu(a), a)$, we present the numerical solution to the equation $F(\mu(a), a)$ $=0$ (18) in tabulated form.

It follows from the table that the solution to this equation is the function $\mu(a)$ which monotonically weakly varies within the limits $[20-22]$ :

$$
0.304 \leq \mu(a) / m_{0} \leq 0.359
$$

as the QD radius $a$ varies within the range

$$
2.0 \leq a \leq 29.8 \mathrm{~nm}
$$


( $m_{0}$ is the electron mass in vacuum). In this case, the reduced exciton effective mass $\mu(a)$ (19) in the nanosystem slightly differs from the effective mass of an exciton (with a spatially separated hole and electron) $\mu=0.304 m_{0}$ by the value $\left(\mu(a)-\mu_{0}\right) / \mu_{0} \leq 0.18$ when the QD radii vary within the range (20).

Substituting the values of the variational parameter $\mu(a)$ (19) from the table simultaneously with the corresponding QD radii from the range (20) into the functional $E_{0}(a, \mu(a))$ (17), we obtain the exciton ground-state energy $E_{0}(a, \varepsilon)(17)$ as a function of the QD radius $a$. [20 - 22].

The results of variational calculation of the energy of the ground state of an exciton $E_{0}(a, \varepsilon)(17)$ in the nanosystem under study containing zinc-selenide QDs of the radius $a$ (20) are shown in the figure [20 - 22]. Here, the values of function $\mu(a)$ (19) and the results of variational calculation of the exciton ground-state energy $E_{0}(a, \varepsilon)$ (17) are obtained for a nanosystem containing zinc-selenide QDs, synthesized in a borosilicate glass matrix, studied in the experimental works $[10,19]$.

In the experimental work [10], borosilicate glass samples doped with zinc selenide with concentrations from $x=$ 0.003 to $1 \%$, obtained by the sol-gel method were studied. According to X-ray diffraction measurements, the average radii $a$ of $\mathrm{ZnSe}$ QDs formed in the samples are within $a \approx$ $2.0-4.8 \mathrm{~nm}$.. In this case, the values of $\tilde{a}$ are comparable to the exciton Bohr radius $a_{e x} \approx 3.7 \mathrm{~nm}$ in a zinc-selenide single crystal. At low QD concentrations $(x=0.003$ and $0.06 \%$ ), their interaction can be disregarded. The optical properties of such nanosystems are mainly controlled by the energy spectra of electrons and holes localized near the spherical surface of individual QDs synthesized in the borosilicate glass matrix.

In $[10,19]$, a peak in the low-temperature luminescence spectrum at an energy of $E 1 \approx 2.66 \mathrm{eV}$ was observed at the temperature $T=4.5 \mathrm{~K}$ in samples with $x=0.06 \%$; this energy is lower than the band gap of a zinc-selenide single crystal $\left(E_{g}=2.823 \mathrm{eV}\right)$. The shift of the peak of the lowtemperature luminescence spectrum with respect to the band gap of the $\mathrm{ZnSe}$ single crystal to the short-wavelength region is $\Delta E 1=\left(E 1-E_{g}\right) \approx-165 \mathrm{meV}$. The authors of [10] assumed that the shift $\Delta E 1$ is caused by quantum confinement of the energy spectra of electrons and holes localized near the spherical surface of individual QDs and is associated with a decrease in the average radii $a$ of zincselenide QDs at low concentrations $(x=0.06 \%)$. In this case, the problem of the quantum confinement of which electron and hole states (the hole moving within the QD volume and the electron localized at the outer spherical QD-dielectric matrix interface or the electron and hole

$$
\begin{gathered}
\left(\bar{V}_{e e^{\prime}}(a, \mathcal{E})+\bar{V}_{h h^{\prime}}(a, \mathcal{E})+\bar{V}_{e h^{\prime}}(a, \mathcal{E})+\bar{V}_{h e^{\prime}}(a, \mathcal{E})\right)= \\
=\left\langle\psi_{\mathrm{o}}\left(r_{e}, r_{h}, r, a\right)\left|V_{e e^{\prime}}\left(r_{e}, a, \mathcal{E}\right)+V_{h h}\left(r_{h}, a, \mathcal{E}\right)+V_{e h^{\prime}}\left(r_{e}, r_{h}, a, \mathcal{E}\right)+V_{h e^{\prime}}\left(r_{e}, r_{h}, a, \mathcal{\varepsilon}\right)\right| \psi_{\mathrm{o}}\left(r_{e}, r_{h}, r, a\right)\right\rangle
\end{gathered}
$$

makes a significantly smaller contribution to the exciton ground-state energy (17), $0.04 \leq \mid\left[V_{e e^{\prime}}(a, \varepsilon)+V_{h h^{\prime}}(a, \varepsilon)+\right.$

$$
\left.V_{e h^{\prime}}(a, \varepsilon)+V_{h e^{\prime}}(a, \varepsilon)\right] /(a, \varepsilon) \mid \leq 0.12[20-22] \text {. }
$$

localized in the QD volume) caused such a shift of the luminescence-spectrum peak remained open.

Comparing the exciton ground-state energy $\left(E_{0}(a, \varepsilon)-E_{g}\right)$ (17) with the energy of the shift in the luminescencespectrum peak $\Delta E 1 \approx-165 \mathrm{meV}$, we obtain the average zincselenide QD radius $a 1 \approx 4.22 \mathrm{~nm}$ (see the figure) [20 - 22]. The QD radius $a 1$ may be slightly overestimated, since variational calculation of the exciton ground-state energy can give slightly overestimated energies [33, 34]. The determined average QD radius $a 1$ lies within the range of the average radii of zinc-selenide QDs $(a \approx 2.0-4.8 \mathrm{~nm})$, studied under the experimental conditions of $[10,19]$.

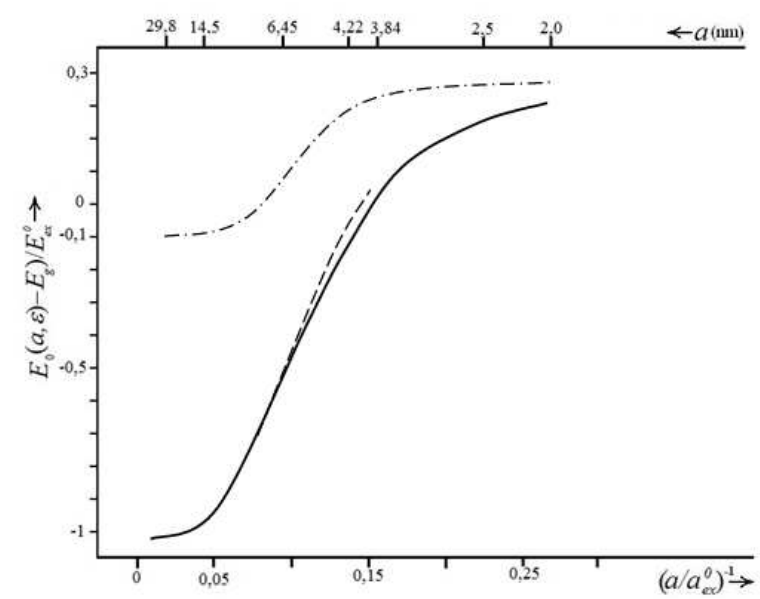

Figure 1. Dependences of the exciton ground state-energy $\left(E_{0}(a, \varepsilon)-E_{g}\right)$ (17) (solid curve) and the binding energy of the exciton ground state $\left(E_{e x}(a, \varepsilon)-E_{g}\right)(21)$ (dashed curve) on the zinc-selenide $Q D$ radius a in the model of an exciton with a spatially separated electron and hole. The dash-dotted curve is the dependence of the exciton ground-state energy $\left(E_{0}(a, \varepsilon)-E_{g}\right)$ on the zinc-selenide $Q D$ radius $a$ in the exciton model, in which the electron and hole move within the zinc-selenide $Q D$ volume [16]. Eg $=2.823 \mathrm{eV}$ is the band gap in a zinc-selenide single crystal; $E_{e x}^{0}$ $=1.5296 \mathrm{eV}(15)$ and $a_{e x}^{0},=0.573 \mathrm{~nm}$ (14) are, respectively, the binding energy of the ground state and the Bohr radius of a two-dimensional exciton with a spatially separated electron and hole.

It should be noted that the average Coulomb interaction energy $\overline{V_{e h}}(a, \varepsilon)=\left\langle\psi_{0}\left(r_{e}, r_{h}, r, a\right)\left|V_{e h}(r)\right| \psi_{0}\left(r_{e}, r_{h}, r, a\right)\right\rangle$, between the electron and hole mainly contribute to the ground-state energy (17) of the exciton in the nanosystem containing zinc-selenide QDs with radii $a 1$ comparable to the exciton Bohr radius in a zinc- selenide single crystal $\left(a_{e x}\right.$ $\approx 3.7 \mathrm{~nm}$ ). In this case, the average energy of the interaction of the electron and hole with self- and "foreign" images,
Thus, the short-wavelength shift $\Delta E 1$ of the low temperature luminescence spectrum peak is caused by renormalization of the electron-hole Coulomb interaction energy $V_{e h}(r)(11)$ and also renormalization of the energy $U\left(r_{e}, r_{h}, r, a, \varepsilon\right)(5)$ of the polarization interaction of the 
electron and hole with the spherical QD-dielectric matrix interface, which is associated with spatial confinement of the quantization region by the QD volume. In this case, the hole moves within the QD volume, and the electron is localized at the outer spherical QD-dielectric matrix interface.

\subsection{Binding Energy of the Exciton Ground State in the Nanosystem}

The binding energy of the ground state of an exciton (with a spatially separated electron and hole) $E_{e x}(a, \varepsilon)$ in a nanosystem containing zinc-selenide QDs of the radius $a$ is the solution to the radial Schrodinger equation with a Hamiltonian containing, in contrast to Hamiltonian (10), only the terms $V_{h e^{\prime}}\left(r_{e}, r_{h}, a, \varepsilon\right)(8)$ and $V_{e h^{\prime}}\left(r_{e}, r_{h}, a, \varepsilon\right)(9)$ in the polarization interaction energy $U\left(\mathrm{r}_{e}, \mathrm{r}_{h}, a, \varepsilon\right)(5)$, which describe the energies of the hole and electron interaction with "foreign" images, respectively [15, 27, 28]. Therefore, the exciton ground-state binding energy $\operatorname{Eex}(a, \varepsilon)$ is defined by the expression $[20-22]$ :

$$
E_{e x}(a, \mathcal{E})=E_{0}(a, \mathcal{E})-\left\langle\psi_{\mathrm{o}}\left(r_{e}, r_{h}, r, a\right)\left|\left(V_{h h^{\prime}}\left(r_{h}, a, \mathcal{E}\right)+V_{e e^{\prime}}\left(r_{e}, a, \mathcal{E}\right)\right)\right| \psi_{\mathrm{o}}\left(r_{e}, r_{h}, r, a\right)\right\rangle,
$$

Where the term

$\left\langle\psi_{\mathrm{o}}\left(r_{e}, r_{h}, r, a\right)\left|\left(V_{h h^{\prime}}\left(r_{h}, a, \mathcal{E}\right)+V_{e e^{\prime}}\left(r_{e}, a, \mathcal{E}\right)\right)\right| \psi_{\mathrm{o}}\left(r_{e}, r_{h}, r, a\right)\right\rangle$

describes the average energy of hole and electron interaction with self-images.

Since the average energies of the interaction of the hole with its image and the average energies of the interaction of the electron with its image make contri-butions with opposite signs to expression (21), they significantly compensate each other. Therefore, the binding energies of the exciton ground state $E_{e x}(a, \varepsilon)(21)$ slightly differ from the corresponding total energies of the exciton ground state $E_{0}(a, \varepsilon)(17)$. This difference

$$
\Delta=\mid\left(E_{e x}(a, \mathcal{\varepsilon})-E_{0}(a, \mathcal{\varepsilon})\right) / E_{e x}(a, \varepsilon),
$$

varies within $\Delta \leq 4 \%$ as QD radii $a$ vary within the range $3.84 \leq a \leq 8.2 \mathrm{~nm}$ (see the figure) [20 - 22].

The figure shows the dependences of the total energy $E_{0}(a, \varepsilon)(17)$ and the binding energy $E_{e x}(a, \varepsilon)(21)$ of the ground state of the exciton with a spatially separated electron and hole on the QD size for a nanosystem containing zinc-selenide QDs of the radius $a$. We can see that bound states of electron-hole pairs arise near the spherical surface of the QD starting from the QD critical radius $a \geq a_{c}^{(1)} \approx 3.84 \mathrm{~nm}$. In this case, the hole is localized near the QD inner surface, and the electron is localized at the outer spherical QD-dielectric matrix interface. Starting from the QD radius $a \geq a_{c}^{(1)}$, the electron-hole pair states are in the region of negative energies (counted from the top of the band gap $E_{g}$ for a zinc-selenide single crystal), which corresponds to the electron-hole bound state [20 -22, $29-$ 23]. In this case, the electron-hole Coulomb interaction energy $V_{e h}(r)(11)$ and the energy $U\left(r_{e}, r_{h}, r, a, \varepsilon\right)$ (5) of the polarization interaction of the electron and hole with the spherical QD-dielectric matrix interface dominate over the energy of quantum confinement of the electron and hole in the nanosystem under study.

The total energy $\left|E_{0}(a, \varepsilon)\right|(17)$ and the binding energy $\left|E_{e x}(a, \varepsilon)\right|(21)$ of the ground state of the exciton with a spatially separated electron and hole increase with QD radius $a$. In the range of radii

$$
4.0 \leq a \leq 29.8 \mathrm{~nm}
$$

the binding energy $\left|E_{e x}(a, \varepsilon)\right|(21)$ of the exciton ground state significantly (by a factor of 4.1-76.2) exceeds the

exciton binding energy in a zinc-selenide single crystal, $\overline{E_{e x}^{0}} \approx-21.07 \mathrm{meV}$ Starting from the QD radius $a \geq a_{c}^{(2)} \approx$ $29.8 \mathrm{~nm}$, the total energies (17) and binding energies (21) of the exciton ground state asymptotically tend to the value $E_{e x}^{0}=-1.5296 \mathrm{eV}$ which characterizes the binding energy of the ground state of a two-dimensional exciton with a spatially separated electron and hole (see the figure) [20 22, $29-32]$.

The obtained values of the total energy $E_{0}(a, \varepsilon)(17)$ of the exciton ground state in the nanosystem satisfy the inequality

$$
\mathrm{E} 0(\mathrm{a}, \varepsilon)-\mathrm{Eg} \ll \Delta V(a)
$$

where $\Delta V(a)$ is the potential-well depth for the QD electron For a large class of II-VI semiconductors in the region of QD sizes $a \geq a_{e x}^{0}, \Delta V(a)=2.3-2.5 \mathrm{eV}$ [7]. Satisfaction of condition (23), probably, makes it possible to disregard the effect of the complex structure of the QD valence band on the total energy (17) and the binding energy (21) of the exciton ground state in the nanosystem under study when deriving these quantities.

The effect of a significant increase in the binding energy $\left|E_{e x}(a, \varepsilon)\right|(21)$ of the exciton ground state in the nanosystem under study, according to formulas (5)-(9), (11), (13)-(15), (17), (21) is controlled by two factors [20- 22, $29-32]$ : (i) a significant increase in the energy of the electron-hole Coulomb interaction $\left|V_{e h}(r)\right|(11)$ and an increase in the energy of the interaction of the electron and hole with "foreign" images $\left|V_{e h^{\prime}}\left(r_{e}, r_{h}, r, a, \varepsilon\right)\right|(9),\left|V_{h e^{\prime}}\left(r_{e}, r_{h}, r, a, \varepsilon\right)\right|$ (8) (the "dielectric enhancement" effect [34]); (ii) spatial confinement of the quantization region by the QD volume; in this case, as the QD radius $a$ increases, starting from $a$ $\geq a_{c}^{(2)} \approx 52 a_{e x}^{0} \approx 29.8 \mathrm{~nm}$, the exciton becomes twodimensional with a ground- state energy $E_{\text {ex }}^{0}$ (15) that exceeds the exciton binding energy $E_{e x}$ in the zincselenide single crystal by almost two orders of magnitude $\left(\left|E_{\text {ex }}^{0} / \overline{E_{e x}^{0}}\right| \approx 72.6\right)$.

The "dielectric enhancement" effect is caused by the following factor. When the matrix permittivity $\varepsilon_{1}$ is significantly smaller than the QD permittivity $\varepsilon_{2}$, the most important role in the electron-hole interaction in the nanosystem under study is played by the field induced by these quasiparticles in the matrix. In this case, electronhole interaction in the nanosystem appears to be significantly stronger than in an infinite semiconductor 
with the permittivity $\varepsilon_{2}[34]$.

In [16], in the nanosystem experimentally studied in [10], an exciton model in which the electron and hole move within the zinc-selenide QD volume is studied. Using the variational method, within the modified effective mass method, the dependence of the exciton ground-state energy $E_{0}(a, \varepsilon)$ on the QD radius $a$ in the range (20) was obtained in [16] (see the figure). It was shown that, as the QD radius increases, starting from $a \geq a_{c}=3.90 \overline{a_{e x}^{0}}=1.45 \mathrm{~nm}$, a bulk exciton appears in the QD; its binding energy

$$
\overline{E_{e x}^{0}}=-\frac{\hbar^{2}}{2 \mu\left(\overline{a_{e x}^{0}}\right)^{2}}
$$

is $21.07 \mathrm{meV}\left(\mu=0.132 m_{0}\right.$ and $\overline{a_{e x}^{0}}=3.7 \mathrm{~nm}$ are the reduced effective mass and Bohr radius of the exciton in the zinc selenide forming the QD volume). The bulk exciton in the QD is understood as an exciton whose structure (reduced mass, Bohr radius, and binding energy) in the QD does not differ from the structure of an exciton in an infinite semiconductor material. As the QD radius $a$ increases $\left(a \geq a_{c}\right)$, the exciton ground-state energy $E_{0}(a)$ asymptotically follows the binding energy of the bulk exciton (24) (see the figure) [20 - 22, $29-32]$.

Thus, using the exciton model in which an electron and hole move in the QD volume, it is impossible to interpret the mechanism of the appearance of the nanosystem luminescence - spectrum peak with the shift $\Delta E 1 \approx-165$ $\mathrm{meV}$, obtained in $[10,19]$.

A comparison of the dependences of the exciton groundstate energy $E_{0}(a)$ in the nanosystem [10], obtained using two - exciton models (see the figure) (the electron and hole move within the zinc-selenide QD volume [16] (model I); the hole moves within the zinc-selenide QD volume, and the electron is localized in the boron silicate glass matrix near the QD spherical surface (model II) allows the following conclusion. In model $\mathrm{I}$, as the QD radius $a$ increases, starting from $a \geq a_{c} \approx 14.5 \mathrm{~nm}$, the exciton ground- state energy $E_{0}(a)$ asymptotically follows the binding energy of the bulk exciton $\overline{E_{e x}^{0}} \approx-21.07 \mathrm{meV}$ (24); in model II, as the QD radius increases, starting from $a$ $\geq a_{c}^{(2)} \approx 29.8 \mathrm{~nm}$, the exciton ground- state energy (17) asymptotically follows $E_{e x}^{0}=-1.5296 \mathrm{eV}$ (15) (characterizing the binding energy of the ground state of a two- dimensional exciton with a spatially sep arated electron and hole), which is significantly lower than $\overline{E_{e x}^{0}} \approx-$ $21.07 \mathrm{meV}$ [20- 22, 29 - 32].

\section{Excitonic Quasimolecules Formed from Spatially Separated Electrons and Holes}

We consider a model nanosystem $[23,24]$ that consists of two spherical semiconductor QDs, $A$ and $B$, synthesized in a borosilicate glass matrix with the permittivity $\varepsilon_{1}$. Let the QD radii be $a$, the spacing between the spherical QD surfaces be $D$. Each QD is formed from a semiconductor material with the permittivity $\varepsilon_{2}$. For simplicity, without loss of generality, we assume that the holes $h(A)$ and $h(B)$ with the effective masses $m_{h}$ are in the QD (A) and QD (B) centers and the electrons $e(1)$ and $e(2)$ with the effective masses $m_{e}^{(1)}$ are localized near the spherical $\mathrm{QD}(\mathrm{A})$ and QD (B) surfaces, respectively. The above assumption is reasonable, since the ratio between the effective masses of the electron and hole in the nanosystem is much smaller that unity: $\left(\left(m_{e}^{(1)} / m_{h}\right) \ll 1\right)$. Let us assume that there is an infinitely high potential barrier at the spherical QD matrix interface. Therefore, in the nanosystem, holes do not leave the QD bulk, whereas electrons do not penetrate into the QDs.

In the context of the adiabatic approximation and effective mass approximation, using the variational method, we obtain the total energy $E_{0}(\widetilde{D}, \tilde{a})$ and the binding energy $E_{b}(\widetilde{D}, \widetilde{a})$ of the biexciton singlet ground state (the spins of the electrons $e(1)$ and $e(2)$ are antiparallel) in such system as functions of the spacing between the QD surfaces be $D$ and the QD radius $a[23,24]$ :

$$
E_{0}(\widetilde{D}, \tilde{a})=2 E_{e x}(\tilde{a})+E_{\mathrm{B}}(\widetilde{D}, \tilde{a})
$$

Here, the binding energy $E_{e x}(\tilde{a})$ (17) of the ground state of the exciton (formed from an electron and a hole spatially separated from the electron) localized above the QD(A) (or $\mathrm{QD}(\mathrm{B}))$ surface is determined by in [23, 24] (parameters $\tilde{a}=\left(a / a_{e x}^{0}\right)\left(a_{e x}^{0}=3,7 \mathrm{~nm}-\right.$ the exciton Bohr radius in a single crystal $\left.\mathrm{ZnSe}, \widetilde{D}=\left(D / a_{e x}^{0}\right)\right)$. For the nanosystem under study, the values of the binding energies $E_{\text {ex }}(\tilde{a})$ are calculated in $[23,24]$ for the experimental conditions of $[10$ 19].

The results of variational calculation of the binding energy $E_{\mathrm{B}}(\widetilde{D}, \widetilde{a})$ of the biexciton singlet ground state in the nanosystem of $\mathrm{ZnSe}$ QDs with average radii of $\bar{a}_{1}=$ $3,88 \mathrm{~nm}$, synthesized in a borosilicate glass matrix are shown in [23, 24]. Such a nanosystem was experimentally studied in [10, 19]. In [10, 19], the borosilicate glassy samples doped with $\mathrm{ZnSe}$ to the content $x$ from $x=0.003$ to $1 \%$ were produced by the sol-gel technique. At a QD content of $x=0.06 \%$, one must take into account the interaction of charge carriers localized above the QD surfaces.

The binding energy $E_{\beta}(\widetilde{D}, \widetilde{a})$ of the biexciton singlet ground state in the nanosystem of $\mathrm{ZnSe}$ QDs with average radii of $\bar{a}_{1}=3,88 \mathrm{~nm}$ has a minimum of $E_{\mathrm{B}}^{(1)}\left(D_{1}, \bar{a}_{1}\right) \approx$ $-4,2 \mathrm{meV}$ (at the spacing $D_{1} \cong 3,2 \mathrm{~nm}$ ) [23, 24]. The value of $E_{\mathrm{B}}^{(1)}$ corresponds to the temperature $T_{\mathrm{c}} \approx$ $49 \mathrm{~K}$ ). In $[23,24]$, it follows that a biexciton (excitonic quasimolecule) is formed in the nanosystem, starting from a spacing between the QD surfaces of $D \geq D_{c}^{(1)} \cong$ $2,4 \mathrm{~nm}$. The formation of such a excitonic quasimolecule (biexciton) is of the threshold character and possible only in a nanosystem with QDs with average radii $\bar{a}_{1}$ such that the spacing between the QD surfaces $D$ exceeds a certain critical spacing $D_{c}^{(1)}$. Moreover, the exciton quasimolecule (biexciton) can exist only at temperatures below a certain critical temperature: $T_{\mathrm{c}} \approx 49 \mathrm{~K}[23,24]$.

As follows from the results of variational calculation [23, 
24], the binding energy of an exciton (formed from an electron and a hole spatially separated from the electron) localized above the surface of the $\mathrm{QD}(\mathrm{A})$ (or a $\mathrm{QD}(\mathrm{B})$ ) with an average radius of $\bar{a}_{1}=3,88 \mathrm{~nm}$ is $E_{\text {ex }}\left(\bar{a}_{1}\right) \cong$ $-54 \mathrm{meV}$. In this case the energy of the biexciton singlet ground state $E_{0}(\widetilde{D}, \tilde{a})(25)$ takes the value $E_{0}(\widetilde{D}, \widetilde{a})=$ $112 \mathrm{meV}$.

From the results of variational calculation [23, 24], of the biexciton (exciton quasimolecule) binding energy $E_{b}(\widetilde{D}, \tilde{a})$, it follows that the major contribution to the binding energy (25) is made by the average energy of the exchange interaction of the electrons $e(1)$ and $e(2)$ with the holes $h(A)$ and $h(B)$. At the same time, the energy of Coulomb interaction makes a much smaller contribution of the biexciton binding energy $E_{\beta}(\widetilde{D}, \widetilde{a})(25)$.

The major contribution to the exchange - interaction energy is made by the energy of the exchange interaction of the electron $e(1)$ with the holes $h(B)$, as well as of the electron $e(2)$ with the holes $h(B)$, as well as of the electron $e(2)$ with the holes $h(A)$. The major contribution to the Coulomb - interaction energy is made by the energy of Coulomb interaction of the electron $e(1)$ with the holes $h$ $(B)$, as well as of the electron $e(2)$ with the holes $h(A)$ [23, 24].

As the spacing $D$ between the $\mathrm{QD}(\mathrm{A})$ and $\mathrm{QD}(\mathrm{B})$ surfaces is increased, starting from $D \geq D_{c}^{(2)} \cong 16,4 \mathrm{~nm}$, the average Coulomb - interaction energy substantially desreases. In addition, because of the decrease in the overlapping of the electron wavefunction, the average exchange interaction energy substantially decreases as well. As a consequence, the average Coulomb - interaction energy and the average energy of the exchange interaction of the electrons $e(1)$ and $e(2)$ with the holes $h(A)$ and $h(B)$ sharply desrease in comparison with the exciton binding energy $E_{e x}(\tilde{a})(17)[23,24]$, resulting in decomposition of the biexciton in the nanosystem into two excitons (formed of spatially separated electrons and holes) localized above the $\mathrm{QD}(\mathrm{A})$ and $\mathrm{QD}(\mathrm{B})$ surfaces.

\section{New Superatom in the Alkali - Metal Atoms}

For the development of mesoscopic physics and chemistry was essential idea superatoms (or artificial atoms) [20-22,29,30]. Superatom are nanosized quasi-atomic nanostructure formed from spatially separated electrons and holes (the hole in the volume of the QD and the electron is localized on the outer spherical quantum dot matrix dielectric interface) [20-22,29,30]. This terminology may be correct, given the similarity of the spectra of discrete electronic states of atoms and superatomic and the similarity of their chemical activity [20-22,29,30].

In [20-22], in the framework of the modified effective mass method [14], developed the theory of artificial atoms formed from spatially separated electrons and holes (hole moving in the volume of a semiconductor (dielectric) QD and an electron localized on the outer spherical interface between the QD and a dielectric matrix) is developed.
The energy spectrum of superatom (exciton of spatially separated electrons and holes) from QD radius $a \geq a \mathrm{c}$ (about $4 \mathrm{~nm}$ ) is fully discrete [20-22,29,30]. This is called a hydrogen- superatom. It is localized on the surface of a valence electron QD. The energy spectrum of the superatom consists of a quantum-dimension of discrete energy levels in the band gap of the dielectric matrix. Electrons in superatom localized in the vicinity of the nucleus (QD). The electrons moving in well-defined atomic orbitals. Serve as the nucleus of QD containing in its volume semiconductors and insulators. Ionization energy superatoms take large values (of the order of $2.5 \mathrm{eV}$ ), which is almost three orders of magnitude higher than the binding energy of the excitons in semiconductors [2022,29,30].

We briefly discuss the possible physical and chemical effects, which are relevant for the results. In our proposed [20-22,29,30] model of a hydrogen superatom localized on the surface of the QD is a valence electron. In a quasiatomic structures of the outer valence electron can participate in a variety of physical and chemical processes, similar to the atomic valence electrons in atomic structures. Artificial atoms have the ability to connect to their electron orbitals of electrons $N$ (where $N$ can vary from one to several tens). At the same time, the number of electrons $N$ can take values of the order of a few tens or even surpass the serial numbers of all the known elements of Mendeleev's table [20-22,29,30]. This new effect that allows to attach to the electronic orbitals of artificial atoms $N$ electrons causes a high reactivity, and opens up new possibilities superatoms related to their strong oxidizing properties, increasing the possibility of substantial intensity in photochemical reactions during catalysis and adsorption, as well as their ability to form many new compounds with unique properties (in particular, the quasi-molecule and quasicrystals) [24,29,30]. Therefore, studies aimed at a theoretical prediction of the possible existence of artificial new atoms (not listed in the table Mendeleev) and, apparently, in their study of the experimental conditions are very relevant.

Quantum discrete states of the individual atoms of alkali metals are determined by the movement of only one, the outermost valence electron around a symmetric atomic core (containing the nucleus and the remaining electrons) [35]. In the hydrogen superatom formed quantum-energy spectra of discrete energy levels of the valence electron [20$22,29,30]$. Thus, the observed similarity of the spectra of discrete electronic states and individual superatoms alkali metal atoms, and also the similarity of their chemical activity [20-22,29,30,35].

In Section 4, on the basis of analogy spectroscopy of electronic states of artificial atoms and individual alkali metal atoms theoretically predicted a new artificial atom, which is similar to the new alkali metal atom.

\subsection{The new Model of an Artificial Atom}

In [20-22,29,30] proposed a new model of an superatom, which is quasi - zero - dimensional nanosystem consisting of a spherical QD (nucleus superatom ) radius $a$ and that 
includes within its scope semiconductor ( dielectric) with a dielectric constant $\varepsilon_{2}$, surrounded by a dielectric matrix with a dielectric constant $\varepsilon_{1}$. A hole $h$ with the effective mass $m_{h}$ moves in the QD volume, while an electron $e$ with the effective mass $m_{e}{ }^{(1)}$ lies in the dielectric matrix. In such nanostructure lowest electronic level is situated in the matrix and humble hole level is the volume QD. Large shift of the valence band (about $700 \mathrm{meV}$ ) is the localization of holes in the volume QD. Large shift of the conduction band (about $400 \mathrm{meV}$ ) is a potential barrier for electrons (electrons move in the matrix and do not penetrate into the volume QD) . Coulomb interaction energy of an electron and a hole, and the energy of the electron polarization interaction with the surface section (QD - matrix) (since the permittivity $\varepsilon_{2}$ is far superior to QD permittivity $\varepsilon_{1}$ matrix) cause localization of the electron in the potential well above the surface of QD [20-22,29,30 ].

With increasing radius $a \mathrm{QD}$, so that $a \gg a_{e x}{ }^{0}$ (where $a_{e x}{ }^{0}$ (14 ) two-dimensional Bohr radius of the electron) spherical surface section (QD- matrix) transforms into a flat surface section. In this artificial atom electron localized on the surface (QD - matrix) becomes two-dimensional. In this case, the potential energy in the Hamiltonian describing the motion of an electron in superatom, the main contribution to the energy of the Coulomb interaction $V_{e h}(r)$ (11) between an electron and a hole [20-22]. Polarization interaction energy of the electron and hole with a spherical surface section (QD - matrix) gives a much smaller contribution to the potential energy of the Hamiltonian. Thus contribute to a first approximation can be neglected [20-22]. In this regard, the two-dimensional electron energy spectrum $E n$ in the artificial atom takes the form (13).

Dependence of the binding energy $E_{e x}(a, \varepsilon)$ of an electron in the ground state superatom (QD containing zinc selenide radius $a$ and surrounded by a matrix of borosilicate glass [10]), obtained in [20-22] by the variational method, it follows that the bound state of an electron occur near spherical interface (QD-matrix), starting with the value of the critical radius QD $a \geq a_{c}^{(1)}=3.84 \mathrm{~nm}$. When this hole moves in a volume QD, and the electron is localized on the surface of the spherical section (QD - matrix). In this case, the Coulomb interaction energy $V_{e h}(r)(11)$ between the electron and the hole, and the energy of the polarization interaction of electrons and holes with a spherical surface section (QD-matrix) prevail over the size quantization of the energy of electrons and holes in the artificial atom. Thus, in [20-22] found that the occurrence of superatom has a threshold, and is only possible since the radius of QD $\mathrm{KT} a \geq a_{c}^{(1)}=3.84 \mathrm{~nm}$.

With increasing radius of $a$ QD scan, an increase in the binding energy of the electron in the ground state superatom. In the range of radii $4.0 \leq a \leq 29.8 \mathrm{~nm}$ and the binding energy of the electron in the ground state superatom significantly exceeds (in $(4,1-76,2)$ times) the value of the exciton binding energy $\tilde{E}_{e x}^{0} \approx 21.07 \mathrm{meV}$ in a single crystal of zinc selenide [20-22]. Beginning with $a$ radius QD $a \geq a_{c}^{(2)}=29.8 \mathrm{~nm}$, the energy of the ground state of an electron in superatom asymptotically follow the value $E_{e x}^{0}=-1.5296 \mathrm{eV}$, which characterizes the energy of the ground state of two-dimensional electrons in an artificial atom (15) [20-22].

Effect of significantly increasing the energy of the ground state of an electron in superatom mainly determined by two factors [20-22]: 1) a significant increase in the Coulomb interaction energy $\left|V_{e h}(r)\right|(2)$ electron-hole (the "dielectric enhancement" [34]); 2) the spatial limitation on the quantization volume QD, while with increasing radius of $a \mathrm{QD}$, since the radius of QD $a \geq a_{c}^{(2)}=52 a^{0}{ }_{e x}=29.8$ $\mathrm{nm}$ superatomic becomes two-dimensional with a binding energy of the ground state $E_{e x}^{0}(15)$, the value of which is almost two order exceeds the exciton binding energy in a single crystal of zinc selenide. Effect of "dielectric enhancement" due to the fact that when the dielectric constant $\varepsilon_{1}$ of the matrix is much less than the dielectric constant of QD $\varepsilon_{2}$, an essential role in the interaction between the electron and the hole in the superatom playing field produced by these quasi-particles in a matrix. Thus, the interaction between the electron and the hole in the superatom is significantly larger than in a semiconductor permittivity $\varepsilon_{2}$ [34].

\subsection{New Artificial Atom, Which is Similar to a new Single Alkali Metal Atom}

Quantum discrete states of the individual atoms of alkali metals are determined by the movement of only one, the outermost valence electron around a symmetric atomic core (containing the nucleus and the remaining electrons) [35]. At large distances $r$ electron from the nucleus (so that $r \gg$ $a 0$, where $a 0=0.053 \mathrm{~nm}$ - the Bohr radius of the electron in a hydrogen atom), the field of the atomic core is described by the Coulomb field [35]:

$$
V(r)=-\left(Z e^{2} / r\right)
$$

determining the interaction of the valence electron with the atomic core $(\mathrm{Z}$ - serial number of the atom in the periodic table Mendeleev). The energy spectrum of a single atom of an alkali metal hydrogen-described spectrum [35]:

$$
E_{n^{*}}=-\frac{R y^{*}}{\left(n^{*}\right)^{2}}, \quad R y *=Z^{2} R y_{0},
$$

where $n *=(n+y)$ - effective quantum number $(n=1,2$, $3, \ldots$. - the principal quantum number), the amendment $y$ depends on the orbital quantum number $l$. Amendment $y$ in due to the fact that the valence electron moves in the Coulomb field of the atomic core, where the nuclear charge is screened by core electrons. Amendment $y$ correction is determined by comparing the spectrum of (6) with its experimental values. The value of $y<0$, and numerically in the more closer to the atomic core suitable valence electron orbit. The number of possible orbits of the valence electron in a single alkali metal atom such as a hydrogen atom, and [35].

The similarity of the individual series of neutral alkali metal atoms with hydrogen Balmer series suggests that the energy spectra of neutral alkali metal atoms are called valence electron radiation in transitions from higher levels to the level of principal quantum number $n=2$ [35].

In a single atom of an alkali metal valence electron 
moving in the Coulomb field of the atomic core (26) having the same functional dependence on $r$ as the Coulomb field (11), in which the valence electron in hydrogen-like model of artificial atom. This leads to the fact that the energy spectra of the valence electron in a single atom of an alkali metal (27) and in the artificial atom (13) describes the spectrum of hydrogen-type. At the same time, the number of possible quantum states of valence electron in hydrogen-like artificial atom model is the same as the number of quantum states of discrete valence electron in a single atom of an alkali metal [20-22,29,30].

The Table shows the position of the valence electron energy levels in individual atoms of alkali metals $(\mathrm{K}, \mathrm{Rb}$, Sc) [35] and the new artificial atom $X$, as well as the level shifts of the valence electron $\left(\Delta E_{R b}^{\mathrm{K}}, \Delta E_{S c}^{R b}, \Delta E_{x}^{S c}\right)$ relative to the adjacent level. Assume that the shift of the energy level $E_{x}$ artificial atom $\mathrm{X}$ (relative to the energy level $E_{s c}$ of the atom Sc) will be the same as the shift of the energy level $E_{R b}$ of the atom $\mathrm{Rb}$ (relative energy level $E_{s c}$ of the atom Sc), (i.e $\Delta E_{x}^{S c}=\Delta E_{S c}^{R b}$ ). Then the level of the valence electron artificial atom will be $E x=-593 \mathrm{meV}$. Using the dependence of the binding energy $E_{e x}(a, \varepsilon)$ of the ground state of an electron in an artificial atom [20-22] (QD containing zinc selenide radius $a$ and surrounded by a matrix of borosilicate glass [10]), we find the radius QD zinc selenide $a 1=5,4 \mathrm{~nm}$, which corresponds to the $E x=$ $593 \mathrm{meV}$. It should be noted that the energy levels of a valence electron in the individual atoms of alkali metals (K, $\mathrm{Rb}, \mathrm{Sc}$ ) [35] and the new artificial atom $\mathrm{X}$ are located in the infrared spectrum.

Table. Position of energy levels of the valence electron in some alkali metal atoms $(K, R b, S c)$ and a new artificial atom $X$. Level shifts of the valence electron $\left(\Delta E_{R b}^{\mathrm{K}}, \Delta E_{S c}^{R b}, \Delta E_{x}^{S c}\right)$ relative adjacent level.

\begin{tabular}{lll}
\hline $\begin{array}{l}\text { alkali metal } \\
\text { atoms selected }\end{array}$ & $\begin{array}{l}\text { valence electron } \\
\text { energy levels }(\mathrm{meV})\end{array}$ & $\begin{array}{l}\text { level shifts of the } \\
\text { valence electron }(\mathrm{meV})\end{array}$ \\
\hline $\mathrm{K}$ & -721.1 & \\
$\mathrm{Rb}$ & -711.2 & 10 \\
$\mathrm{Sc}$ & -652 & 59 \\
$\mathrm{X}$ & -593 & 59 \\
\hline
\end{tabular}

Thus, we proposed a new model of an artificial atom, which is a quasi-atomic heterostructure consisting of spherical QD (nucleus superatom) radius $a$ and which contains in its scope, zinc selenide, surrounded by a matrix of borosilicate glass (in volume QD moves $h$ hole effective mass $m_{h}, e$ and the electron effective mass $m_{b}{ }^{(1)}$ is located in the matrix), allowed to find a new artificial atom $\mathrm{X}$ (absent in the Mendeleev periodic system), which is similar to a new single alkali metal atom. This new artificial atom of valence electron can participate in various physical [20-22,29,30] and chemicals [30,35] processes analogous atomic valence electrons in atomic systems (in particular, alkali metal atoms selected [35]) . Such processes are unique due to the new properties of artificial atoms: strong oxidizing properties, increasing the possibility of substantial intensity in photochemical reactions during catalysis and adsorption, as well as their ability to form a plurality of the novel compounds with unique properties (in particular, the quasi-molecule and the quasicrystals $[23,24])$.

Application of semiconductor nanoheterostructures as the active region nanolasers prevents small exciton binding energy in QD. Therefore, studies aimed at finding nanoheterostructures, which would be observed a significant increase in the binding energy of the local electronic states in QDs are relevant [20-22]. Effect of significantly increasing the energy of the electron in a hydrogen superatom [20-22,29,30] allows to detect experimentally the existence of such superatoms at room temperatures and will stimulate experimental studies nanoheterostructures containing superatoms that can be used as the active region nanolasers working on optical transitions.

\section{Conclusions}

The theory of an exciton with a spatially separated electron and hole is developed within the modified effective mass method [14] in which the reduced effective exciton mass is a function of the semiconductor QD radius $a$. The average zinc- selenide QD radius was determined by comparing the dependence of the exciton ground-state energy (17) on the QD radius, obtained by the variational method within the modified effective mass method [14], with the experimental peak of the low-temperature luminescence spectrum [10, 19]. It was shown that the short-wavelength shift of the peak of the low-temperature luminescence spectrum of the samples containing zincselenide QDs, which was observed under the experimental conditions of $[10,19]$, is caused by renormalization of the electron-hole Coulomb interaction energy (11) and also the energy of the polarization interaction (5) of the electron and hole with the spherical QD-dielectric matrix interface, related to spatial confinement of the quantization region by the QD volume. In this case, the hole moves in the QD volume, and the electron is localized at the outer spherical QD-dielectric matrix interface [20 -.22, 29 - 32].

To apply semiconductor nanosystems containing zincselenide QDs as the active region of lasers, it is required that the exciton binding energy $\left|E_{e x}(a, \varepsilon)\right|(21)$ in the nanosystem be on the order of several $k T_{0}$ at room temperature $T_{0}$ ( $k$ is the Boltzmann constant) [13]. Nanosystems consisting of zinc- selenide QDs grown in a borosilicate glass matrix can be used as the active region of semiconductor QD lasers. In the range of zinc-selenide QD radii $a(22)$, the parameter $\left|E_{e x}(a, \varepsilon) / k T_{0}\right|$ take significant values in the range from 3.1 to 56 [20 - 22, 29-32].

The effect of significantly increasing the binding energy (21) of the exciton ground state in a nanosystem containing zinc-selenide QDs with radii $a$ (22) was detected; in comparison with the exciton binding energy in a zincselenide single crystal, the increase factor is 4.1-72.6. [20$22,29-32]$. It was shown that the effect of significantly increasing the binding energy (21) of the exciton ground state in the nanosystem under study is controlled by two factors [20 - 22, 29-32]: (i) a substantial increase in the electron-hole Coulomb interaction energy (11) and an 
increase in the energy of the interaction of the electron and hole with "foreign" images (8), (9) (the "dielectric enhancement" effect [34]); (ii) spatial confinement of the quantization region by the QD volume; in this case, as the QD radius $a$ increases, starting from $a \geq a_{c}^{(2)} \approx 29.8 \mathrm{~nm}$, the exciton becomes two-dimensional with a ground-state energy (15) that exceeds the exciton binding energy in a zinc-selenide single crystal by almost two orders of magnitude.

A review devoted to the theory of excitonic quasimolecule (biexciton) (formed of spatially separated electrons and holes) in a nanosystem that consists of ZnSe QDs synthesized in a borosilicate glass matrix is developed within the context of the modified effective mass approximation. Using the variational method, we obtain the total energy and the binding energy of the biexciton singlet ground state in such system as functions of the spacing between the QD surfaces and the QD radius. It is established that, in a nanosystem composed of $\mathrm{ZnSe}$ QDs with the average radii $\bar{a}_{1}$, the formation of a biexciton (exciton quasimolecule) is of the threshold character and possible in a nanosystem, in with the spacing $D$ between the QD surfaces is defined by the condition $D_{c}^{(1)} \leq D \leq D_{c}^{(2)}[23,24]$. Moreover, the exciton quasimolecule (biexciton) can exist only at temperatures below a certain critical temperature: $T_{\mathrm{c}} \approx 49 \mathrm{~K}[23,24]$. It is established that the spectral shift of the low- temperature luminescence peak $[10,19]$ in such a nanosystem is due to quantum confinement of the energy of the biexciton singlet ground state.

Thus, we proposed a new model of an artificial atom, which is a quasi-atomic heterostructure consisting of spherical QD (nucleus superatom) radius $a$ and which contains in its scope, zinc selenide, surrounded by a matrix of borosilicate glass (in volume QD moves $h$ hole effective mass $m_{h}, e$ and the electron effective mass $m_{6}{ }^{(1)}$ is located in the matrix), allowed to find a new artificial atom $\mathrm{X}$ (absent in the Mendeleev periodic system), which is similar to a new single alkali metal atom. This new artificial atom of valence electron can participate in various physical [20$22,29,30]$ and chemicals [30,35] processes analogous atomic valence electrons in atomic systems (in particular, alkali metal atoms selected [35]). Such processes are unique due to the new properties of artificial atoms: strong oxidizing properties, increasing the possibility of substantial intensity in photochemical reactions during catalysis and adsorption, as well as their ability to form a plurality of the novel compounds with unique properties (in particular, the quasi-molecule and the quasicrystals $[23,24])$.

\section{References}

[1] Ekimov, A., Onushchenko, A., (1981). Zize quantization of electrons in microcrystals. Journal Experimental Theoretical Physics Letters. 34, 345-348.

[2] Ekimov, A., Onushchenko, A., (1984). Zize quantization of electrons in semiconductor microcrystals. Journal Experimental Theoretical Physics Letters. 40, 1136-1139.
[3] Ekimov, A., Efros, A., (1985). Excitons in semiconductor microcrystals. Solid State Communication. 56, 921-924.

[4] Ekimov, A., Onushchenko, A., Efros, A., (1986). Zize quantization of electron-hole pairs in nanocrystals. Journal Experimental Theoretical Physics Letters. 43, 376-379.

[5] Chepik, D., Efros, A., Ekimov, A., (1990). Spectroscopy of excitons in semiconductor nanocrystals. Journal of Luminescence. 47, 113-118.

[6] Ekimov, A., Hache, F., Schanne-Klein, M., (2003). Optical properties semiconductor quantum dots. Journal Optical Society American. B 20, $100-108$.

[7] Grabovskis, V., Dzenis, Y., Ekimov, A., (1989). Fotoluminescences of excitons in semiconductor nanocrystals. Physics Solid State. 31, 149 -152.

[8] Alferov, J.I., (2002). Progress development in semiconductor nanostructures. Physics Uspekhi. 172, 1068 1074.

[9] Alferov, J.I., (1998). Optical properties in semiconductor nanostructures. Semiconductors. 32, 1-8.

[10] Bondar, V.N., Brodin, M.S., (2010). Optical properties semiconoductor quantum dots. Semiconductors. $44,884-890$.

[11] Efros, A.L., Efros, Al.L., Interband absorption light in semiconductor sphere. (1982). Sov. Phys. Semiconductors. $16,955-962$.

[12] Pokutnyi, S.I., (2004). Size quantization Stark effect in semiconductor quantum dots. J. Appl. Phys. 96, 1115-1122.

[13] Pokutnyi, S.I., (2005). Optical nanolaser heavy hole transitions in quasi - zero - dimensional semiconductors nanosystems. Physics Lett. A. 342, 347-352.

[14] Pokutnyi, S.I., (2007). Exciton states in semiconductor quantum dots in the framework of the modified effective mass method. Semiconductors. 41, 1323-1331.

[15] Pokutnyi, S.I., (2010). Exciton states in semiconductor quantum dots. Semiconductors. 44, 488-493.

[16] Pokutnyi, S.I., (2012). Exciton states in semiconductor nanosystems. Semiconductors. 46, 174-184.

[17] Soloviev, V., Eeichofer, A., (2001). Approximation of effective mass in nanosystems. Physica Status Solidi B. 224, 285-291.

[18] Yeh, C., Zhang, S., Zunger, A., (2004). The effective mass method in nanosystems. Physical Review B. 62, 1440814416.

[19] Bondar, N., Brodyn, M., (2010). Spectroscopy of semiconbductor quantum dots. Physics E. 42, 1549-1555.

[20] Pokutnyi, S.I., (2013). Binding energy of the exciton of a spatially separated electron and hole in quasi - zero dimensional semiconductor nanosystems. Technical Physics Letters. 39, 233-235.

[21] Pokutnyi, S.I., (2013). On an exciton with a spatially separated electron and hole in quasi - zero - dimensional semiconductor nanosystems. Semiconductors. 47, 791 -798.

[22] Pokutnyi, S.I., (2014). Theory of excitons formed from spatially separated electrons and holes in quasi - zero dimensional semiconductor nanosystems. SOP Transactions Theoretical Physics. 1, 24 -35. 
[23] Pokutnyi, S.I., (2013). Biexcitons formed from spatially separated electrons and holes in quasi - zero - dimensional semiconductor nanosystems. Semiconductors. 47, 1626 1635.

[24] Pokutnyi, S.I., (2014). Quasi - zero - dimensional nanostructures: Excitonic quasimolecules. J. Appl. Chem. 2, 1- 4 .

[25] Efremov, N.A., Pokutny, S.I., (1985). Macroscopic local charge states in ultradispersion media. Sov. Phys. Solid. State. 27, 27-35.

[26] Efremov, N.A., Pokutny, S.I., (1990). Energy spectrum of exciton in spherical particle. Sov. Phys. Solid. State. 32, 955964

[27] Pokutnyi, S.I., (1992). Size quantization of electron-hole pair in semiconductor quantum dots. Physics Lett. A. 168, 433-438.

[28] Pokutnyi, S.I., (2011). Theory of exciton states in semiconductor nanosystems. Physics Express. 1, 158-164.
[29] Pokutnyi, S.I., Gorbyk, P.P., (2013). Superatoms in quasi zero - dimensional nanostructures (review). Progr. Phys. Metal. 14, 144 - 168.

[30] Pokutnyi, S.I., Gorbyk, P.P., (2013). Superatoms in quasi zero - dimensional nanosystems. J. Appl. Chem. 1, 44 - 47.

[31] Pokutnyi, S.I., (2012). Exciton states in quasi - zero dimensional semiconductor nanosystems: Theory. Phys. Express. 2, 20 - 26.

[32] Pokutnyi, S.I., (2013). Exciton states in quasi - zero dimensional nanostructures. J. Appl. Chem. 1, 5 - 18.

[33] Lozovik, Y., Nishanov, V., (1976). Exciton Wannier-Mott near the interface. Physics Solid State. 18, 1905-1911.

[34] Keldysh, L., (1979). The interaction of the electrons in a thin films. Journal Experimental Theoretical Physics Letters. 29, 621-624.

[35] Frish, S.E., (1963). Optical spectra of atoms. Nauka, Moscow [in Russian]. 